WellBeing International

WBI Studies Repository

$10-1975$

\title{
Vocalizations of Isolated Piglets. I. Sources of Variation and Relationships Among Measures
}

D. Fraser

University of Edinburgh

Follow this and additional works at: https://www.wellbeingintlstudiesrepository.org/comski

Part of the Animal Studies Commons, Comparative Psychology Commons, and the Other Animal Sciences Commons

\section{Recommended Citation}

Fraser, D. (1975). Vocalizations of isolated piglets. I. Sources of variation and relationships among measures. Applied Animal Ethology, 1(4), 387-394.

This material is brought to you for free and open access by WellBeing International. It has been accepted for inclusion by an authorized administrator of the WBI Studies Repository. For more information, please contact wbisr-info@wellbeingintl.org.

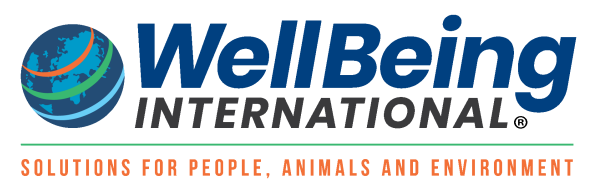




\title{
Vocalizations of Isolated Piglets. I. Sources of Variation and Relationships Among Measures
}

\author{
D. Fraser \\ University of Edinburgh
}

\begin{abstract}
The vocalizations and activity of suckling piglets aged 4 to 25 days were studied during 5min periods of social isolation in an unfamiliar enclosure. The animals became very active and vocalized repeatedly during the test, but the reaction was attenuated among older litters. Calls were predominantly closed mouth grunts at low rates of calling, and mainly squeals and open mouth grunts at high overall rates. Squealing correlated with open mouth grunting and with the activity measure both within and between litters. Even so, the measures appeared to be affected independently by the piglets' behaviour in the home pen just before the test, and by their habits of fighting during suckling. Individual scores for squealing and open mouth grunting correlated with scores for the same measures on a subsequent re-test. Relationships among the types of vocalization, and individual differences among piglets in their reactions to the test, were discussed.
\end{abstract}

\section{INTRODUCTION}

When a suckling piglet is removed to an unfamiliar enclosure away from its dam and littermates, it generally gives a characteristic pattern of loud, repeated vocalizations coupled with vigorous activity. In more natural surroundings this unstoical behaviour would probably help to reunite an errant piglet with its dam. The reaction is of interest in modem animal husbandry partly because it appears to be an expression of "distress". Analysis of the various aspects of the reaction might; therefore, provide general information of use in determining causes and symptoms of thwarting or discomfort among piglets. The reaction is also of interest because of its basic similarity to the initial response of many artificially-reared piglets to their new environment after weaning. The ease with which early-weaned piglets adapt to a particular system of management may well be reflected in the speed with which their initial heightened activity and vocalizing decline.

In the following experiment, activity and vocalizations were studied among piglets removed briefly from the maternal environment. Causes of variation in their behaviour were analysed both within and between litters, and relationships among the different measures were examined. 


\section{METHOD}

18 litters of piglets were chosen from a herd of Large White (LW) and Landrace (Lr) stock. The litters consisted of 6 to 11 young giving a total of 159 piglets. When the experiment was conducted there were three main classes of breeding dams in the herd: 1st parity LW females, 2nd parity LW females, and 1st parity LW $\times$ Lr females. Each litter was tested at one of six ages - 4, 8,10,12,18, and 21 to 25 days - and was the offspring of one of the three classes of dam.

The pigs were tested in a featureless pen measuring $1.8 \times 2.4 \mathrm{~m}$ with wooden walls $1.1 \mathrm{~m}$ high. Two walls were topped with glass windows which sloped inward, while another had a solid, hinged door. The concrete floor was divided into six rectangular sections of equal size. The pen was equipped with a suspended lamp and a microphone which led to a tape recorder. An exhaust fan provided uniform background noise. The pen was situated in a room which contained no animals but was in the same building as the accommodation for sows and litters.

The piglets of a litter were tested individually with as little disturbance as possible to the other littermates. One animal was removed by hand from the home pen after a note had been made of its behaviour just before being taken. The animal was carried in a metal cage from which it was pushed gently into the testing enclosure. For the next $5 \mathrm{~min}$ the tape recorder was turned on, and written notes were made of the number of floor sections the animal entered per minute with all four hoofs inside the section's boundaries. The piglet was then weighed, identified by its ear number, examined for evidence of facial lacerations, and was marked with a coloured number on its back before being returned to the home pen. The litter was typically observed for 2 min before the next piglet was taken for testing, and the time of any suckling activity by the litter was noted. For 12 litters the teat order (i.e., the habitual arrangement of the piglets on the udder during suckling) was noted as described by Fraser and Jones (1975).

In analysing the tape recordings, three types of call were distinguished and counted in each minute of the test. Calls which contained a high-pitched, tonal component were collectively termed "squeals". Previous observations indicated that low-pitched, non-tonal calls contained a vowel sound (e.g., "ma, ma") if made with the mouth open, but contained no vowel sound (e.g., "mm, mm") if made with the mouth closed. Accordingly "open mouth grunts" and "closed mouth grins" were distinguished by ear on this basis. Much more detailed classifications of piglet calls are given by Grauvogl (1958) and by Kiley (1972) who provides a sonographic description of the vocalizations. The three categories used in the present study undoubtedly ignore many of the finer distinctions which can be drawn.

Four of the litters were re-tested in the same way 2 or 3 days after the initial test. A fifth litter was retested according to a similar procedure, but in a smaller wood and wire mesh enclosure, $480 \times 600 \times 480 \mathrm{~mm}$ high, where the animals' mouth movements could be observed closely. The measure of the number of floor sections entered could not be made in this enclosure.

At the conclusion of the experiment seven additional litters, each of which included several piglets with distinct facial lacerations, were tested in the small enclosure in a similar way.

\section{RESULTS}

All the piglets walked or ran about the enclosure, vocalizing frequently. They entered between 16 and 79 floor sections in the $5 \mathrm{~min}$. Several animals jumped against the walls or pushed their snouts against the door in what appeared to be attempts to escape. Closed mouth grunts were given by all piglets; squeals were given by $90 \%$, and open mouth grunts by $39 \%$ of the animals. The number of floor sections entered and the number of closed mouth grunts given in each minute tended to remain constant throughout the 5- 
min exposure, while the frequency of squeals and open mouth grunts generally increased with time, particularly over the first 2 min.

TABLE 1. Correlations among the four measures: Spearman rank order correlation coefficients relating the mean scores per litter for the four measures (above), and mean values of the Pearson correlation coefficient calculated for each litter, relating the scores of individual littermate piglets on the different measures (below)

\begin{tabular}{|lcccc|}
\hline Category & $\begin{array}{c}\text { Closed mouth } \\
\text { grunts }\end{array}$ & $\begin{array}{c}\text { Open mouth } \\
\text { grunts }\end{array}$ & Squeals & $\begin{array}{c}\text { Floor sections } \\
\text { entered }\end{array}$ \\
\hline$r_{s}$ between litter means & ---- & +0.13 & +0.08 & +0.20 \\
Closed mouth grunts & ---- & $+0.88^{\star \star \star}$ & $+0.71^{\star \star \star}$ \\
Open mouth grunts & & --- & $+0.64^{\star \star \star}$ \\
Squeals & & & --- \\
Floor sections entered & & $-0.59^{\star \star \star}$ & +0.08 & \\
Mean $r$ within litters & ---- & $+0.70^{\star \star \star}$ & $+0.24^{\star \star}$ \\
Closed mouth grunts & ----- & -- & +0.18 \\
Open mouth grunts & & & $+0.36^{\star \star \star}$ \\
Squeals & & & ---- \\
Floor sections entered & & &
\end{tabular}

${ }^{\star} P<0.05 ;{ }^{\star \star} P<0.01 ;{ }^{* \star \star} P<0.001$

Fig. 1. The mean number of closed mouth grunts (CMG), open mouth grunts (OMG) and squeals (S) given by individual piglets in relation to the total number of calls of all three types given by the same animals, averaging over the entire experiment. Each point is based on 8 to 22 piglets.

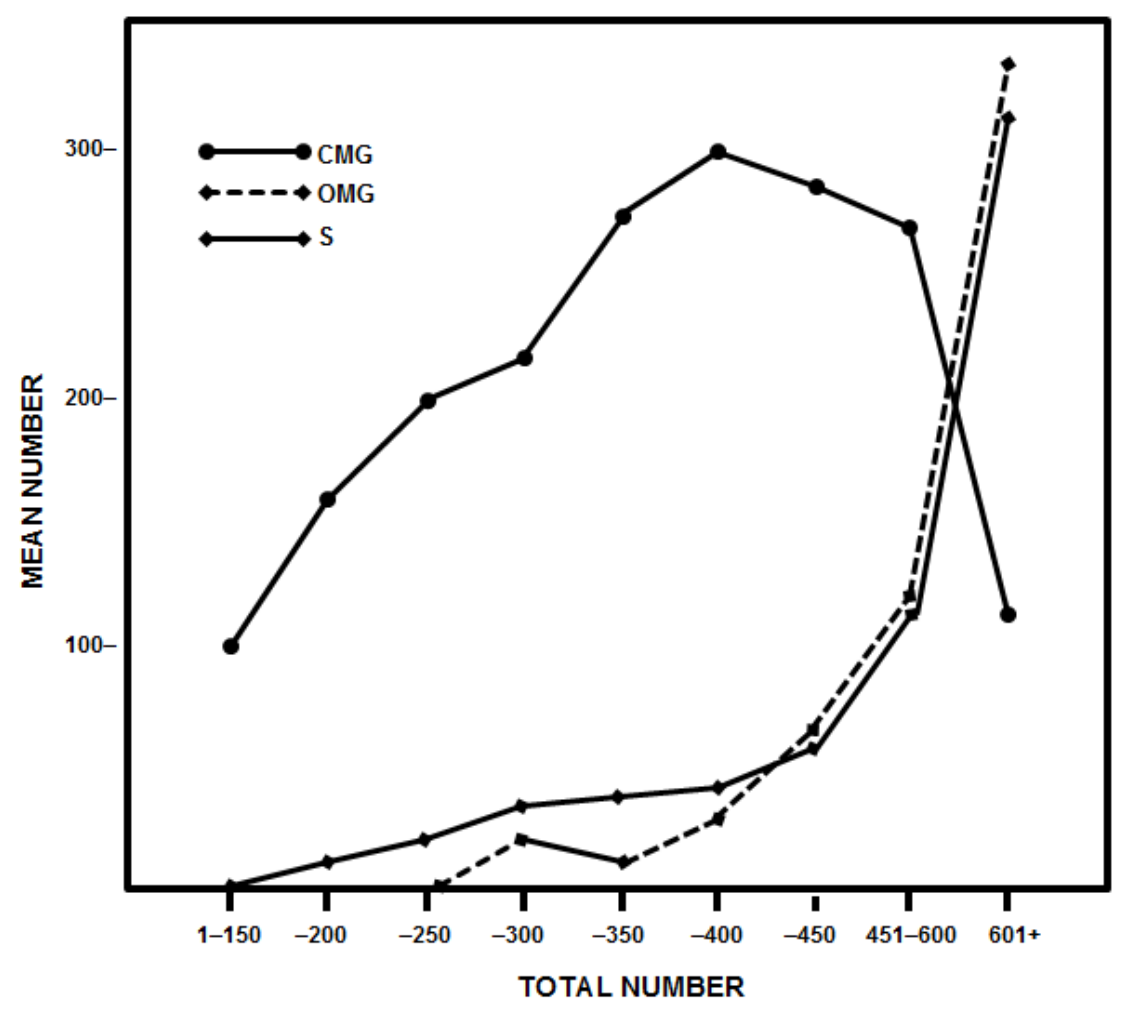


Fig. 2. The mean of the mean scores of the individual litters for the number of floor sections entered (above), and for the number of closed mouth grunts (CMG), open mouth grunts (OMG) and squeals (S) given in the 5min test by litters of different ages.

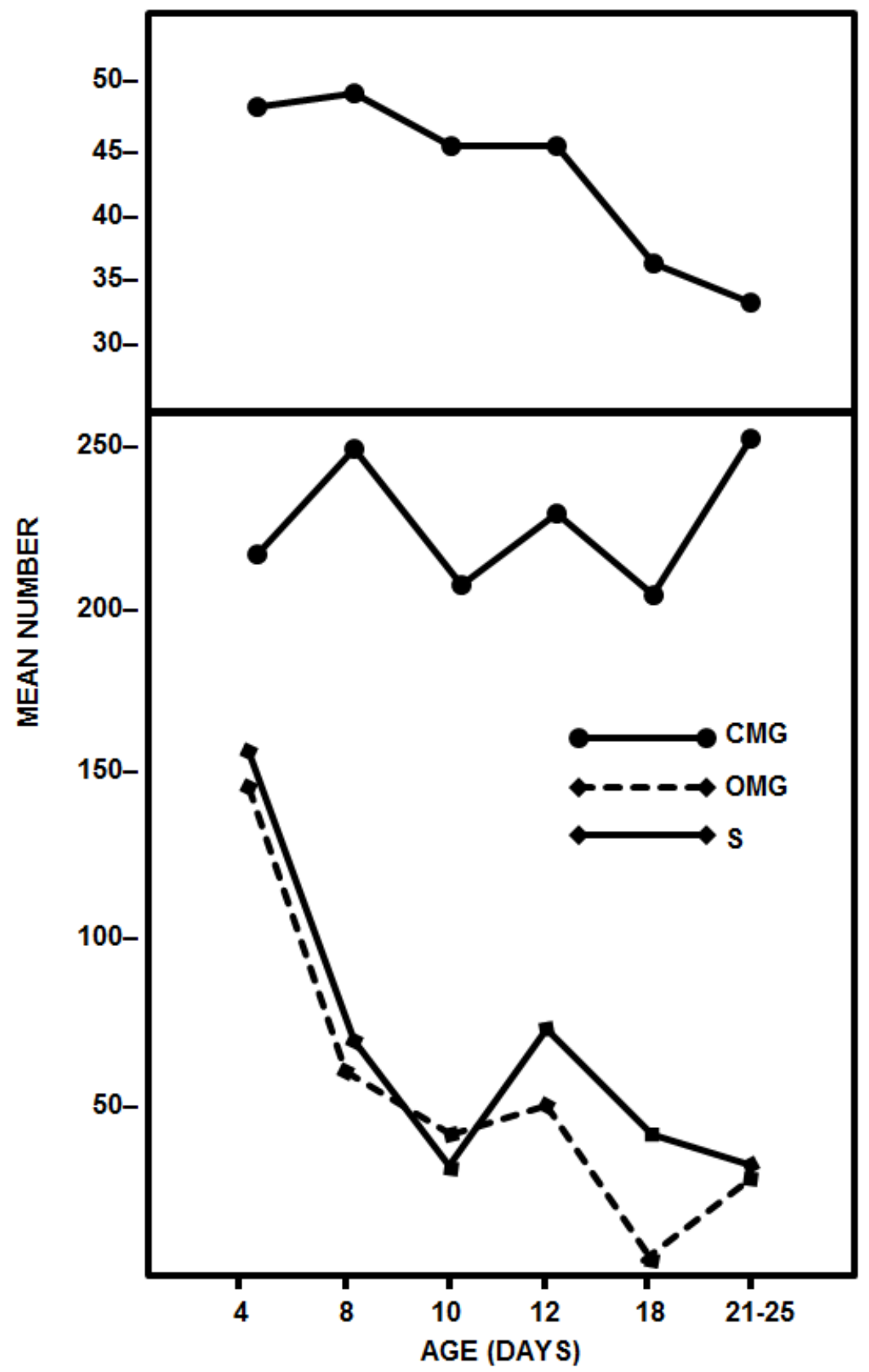

Relationships among measures

The most active litters tended to give the greatest number of squeals and open mouth grunts, while their mean scores for closed mouth grunts were not consistently related to the other measures. The upper part of Table 1 shows the Spearman rank order correlation coefficients for the mean scores of the various litters on the four measures. The non-parametric correlation was used because the litters' mean scores for open mouth grunts did not conform to normal distribution. 
Comparing the performance of individual litter-mate piglets, there was a similar strong association between squeals and both open mouth grunts and floor sections entered, but the latter two measures did not correlate with each other. In addition, piglets with high scores for open mouth grunts tended to have low scores for closed mouth grunts. The lower part of Table 1 shows the mean values of the correlation coefficients relating individual animals' scores on the four measures, averaging the coefficients for each litter. The values of $P$ were calculated by transforming values of the statistics $r$ to $z$.

Relationships among the three vocalizations are further illustrated in Fig.1 which shows the mean number of calls of the three types given by each piglet in relation to the total number of calls the animal gave. Animals with comparatively low rates of calling gave closed mouth grunts almost exclusively, whereas squeals and open mouth grunts predominated among the piglets which called most frequently.

\section{Variation between litters}

The age of the animals was a principal source of variation in behaviour between litters. Fig.2 shows that there was an overall decline with age in the incidence of squeals, open mouth grunts, and floor sections entered, but not closed mouth grunts. Two way analysis of variance for piglet age and the class of the dam was applied to the mean scores per litter. There was a significant effect of age on the average number of squeals $(P<0.05)$, while the effect on the activity measure approached statistical significance $(P<0.10)$. Analysis of variance could not be used in the case of open mouth grunts because the litters' mean scores were not normally distributed. However there was a negative correlation between litter age and mean score for this category $\left(r_{s}=0.72\right.$ corrected for ties, $\left.P<0.01\right)$. Piglets' scores for squealing also varied according to the class of the dam ( $P<0.05$ by analysis of variance). In particular, squealing was more common among the offspring of 1 st parity than of 2 nd parity LW females. There was a similar trend in the scores for open mouth grunts, but not for the other measures.

The number of piglets in the litter did not appear to be related to the animals' performance, except that the four litters of only six or seven piglets had particularly high scores for squealing (140.3 \pm 35.5 , mean \pm S.E. of the litter means) compared with litters of eight or more piglets $(50.4 \pm 8.88)$.

\section{Variation within litters}

For the 12 litters in which the teat order was studied, there was no apparent relationship between the position of the teat pair which a piglet normally occupied and the behaviour of that animal during the test. Similarly an animal's weight at the time of testing did not correlate with its score for closed mouth grunts, open mouth grunts or floor sections entered. However body weight and the incidence of squealing appeared to be related in a manner which depended on the number of piglets in the litter. The mean of the within-litter correlation coefficients relating squealing to body weight was -0.33 for litters of six or seven piglets, and $-0.30,-0.10,+0.04$ and +0.32 for litters of eight, nine, ten and 11 piglets respectively. The values of the coefficients themselves correlated significantly with litter size $\left(r_{s}=+0.56, P=0.01\right)$.

Four of the litters included three or four piglets with dark wounds on their faces, and subsequent observation confirmed that these piglets frequently fought with littermates during suckling. Apart from one wounded piglet which squealed much more than any of its littermates during the test, these animals had particularly low scores for squealing $(28.4 \pm 12.10$, mean \pm S.E., including the one high scoring piglet) compared with their unwounded littermates $(66.4 \pm 14.61)$. This trend was apparent in all four litters, and was also clear in five of the seven additional litters tested subsequently $(P<0.02$ by Student's $t$ test for paired comparisons applied to the mean score of wounded and unwounded piglets in each of the 11 litters). There was no similar relationship between facial wounding and the other behavioural measures. 
Littermate piglets did not appear to differ in their performance depending on when they were tested in relation to times of suckling episodes. However, piglets which were active in the home pen just before testing entered more floor sections than their littermates which had been sleeping before the test. For each litter, median scores were calculated for piglets grouped according to their behaviour in the home pen just before the test. In all 12 litters in which the comparison could be made, the median number of floor sections entered by those which had been sleeping was lower than that of litter-mates which had been active $(P<0.01$ by the sign test), but with the large variation between litters, the range of the medians of the two groups showed considerable overlap.

Piglets which were re-tested after an interval of 2 to 3 days had similar scores for squeals and open mouth grunts on the two occasions. Correlation coefficients were calculated for each litter, relating the individual littermates' scores for the same measures during the two tests. The mean of the within-litter coefficients was +0.43 for squeals and +0.48 for open mouth grunts $(P<0.01$ in both cases), +0.33 for closed mouth grunts and -0.003 for floor sections entered.

\section{DISCUSSION}

The findings help to illustrate the relationships among the different types of piglet vocalizations. At low overall rates of calling, closed mouth grunts and a few squeals were heard. Open mouth grunting began at medium rates of calling, and, together with squealing, appeared gradually to replace closed mouth grunting at very high rates of vocalization. This is consistent in part with the view of Kiley (1972) who suggests that the different types of call form a continuum reflecting what she calls the animal's level of "excitement" as evidenced by its rate of locomotion and frequency of performance of other activities including vocalizations. However Kiley regards the "high grunt" (which apparently corresponds to the open mouth grunt) as intermediate between the basic "common grunt" and the high-pitched, tonal calls. If anything, the reverse is true in the present findings: some tonal calls were given at comparatively low overall rates of vocalization, whereas open mouth grunts began only with higher overall rates. The difficulty in stating which type of call represents the greater degree of agitation of the animal may indicate that it is premature to assume that the different types of call can properly be ranked on a one-dimensional scale. Rather, the transition from closed- to open mouth calls and the transition from low- to high-pitched calls may be affected independently by some factors, such as fighting on the udder as discussed below.

Differences in performance between litters could be linked most clearly to the age of the animals. If the loud calling and vigorous activity of the isolated piglet does represent a means of maintaining contact with the dam, then the decline with age may reflect the growing piglet's increasing tendency toward independence. There was a tendency for squealing and open mouth grunting to be more common among first parity litters, but the sample size was too small for firm conclusions to be drawn. Squealing may also have been influenced by the number of piglets in the litter.

Variation within litters presents a more complicated picture. There was a positive correlation between piglets' original and re-test scores for open mouth grunts and for squeals, suggesting that some of the variation was due to idiosyncratic differences between members of the same litter. However the root of these differences is obscure. There was no simple relationship with the animals' habitual suckling positions or body weight, although squealing may be related to the amount the animals fought at suckling. Fighting during suckling is more common among large litters; it frequently involves facial wounding of the combatants; and in extreme cases habitual fighters may have particularly low body weight (Fraser, 1975). Any tendency for squealing in the test to be depressed by habitual fighting at the udder would help to explain why piglets with facial wounding squealed less than their littermates, why piglets from litters of only six or seven young had high scores for squealing, and why squealing correlated positively with body weight among large litters but not among smaller ones. 


\section{ACKNOWLEDGEMENTS}

This work was supported by the Agricultural Research Council with the co-operation of Professor F.W.H. Elsley and Dr D.G.M. Wood-Gush, and of the pig unit staff.

\section{REFERENCES}

Fraser, D., 1975. The "teat order" of suckling pigs. II. Fighting during suckling and the effect of clipping the eye teeth. J. Apic. Sci., Camb., 84: 393-399.

Fraser, D. and Jones, R.M., 1975. The "teat order" of suckling pigs. I. Relationship to birth weight and subsequent growth. J. Agric. Sci., Camb., 84: 387-391.

Grauvogl, A., 1958. Uber das Verhaiten des Hausschweines mit besonderer Berucksichtigung des Fortpflanzungsverhaltens. Dissertation, Berlin, mimeographed,

Kiley, M., 1972. The vocalizations of ungulates, their causation and function. Z. Tierpsychol., 31: 171-222. 